\section{Implantação do Programa Mais Médicos no Espírito Santo, Brasil: modelo lógico e proposta de indicadores}

\author{
Implementation of the More Doctors Program in \\ Espírito Santo State, Brazil: logic model \\ and proposed indicators
}

\section{Implantación del programa Más Médicos en Espírito Santo, Brasil: modelo lógico y propuesta de indicadores}

\author{
Tatiana Breder Emerich 1 \\ Aline Guio Cavaca 2 \\ Edson Theodoro dos Santos Neto 1 \\ Adauto Emmerich Oliveira 1 \\ Carlos Eduardo Siqueira 3
}

doi: 10.1590/0102-311X00043219

\section{Resumo}

O objetivo foi avaliar a implantação do Programa Mais Médicos (PMM) com base no estudo de caso do Espírito Santo, Brasil. Na primeira etapa, foram analisados os documentos que instituíram o PMM, permitindo a criação de um modelo lógico. Na segunda, foram analisados dados do PMM no Espírito Santo (2013-2016) baseando-se em faixas populacionais, gerando a criação de indicadores da proporção de médicos antes e após o Programa com os parâmetros: inaceitável (0 a 0,99 médico/1.000 habitantes); aceitável (1 a 1,99); e desejável (2,0 ou mais). Os dados foram analisados com os testes não paramétricos de Wilcoxon e Kruskal-Wallis. O modelo incluiu as dimensões, insumos, atividades, produtos, resultados e impacto do PMM, e orientou a análise da implantação do Programa. Do total de médicos para o provimento emergencial para o Espírito Santo (432), a maioria $(63,8 \%)$ pertencia ao perfil intercambistas cooperados (cubanos) e 31,2\% eram médicos com registro no Conselho Regional de Medicina do estado. Tanto os médicos brasileiros quanto os intercambistas individuais foram alocados prioritariamente em municipios de médio e grande portes. Os cubanos se distribuíram de forma mais heterogênea em municípios de todos os portes populacionais e, consequentemente, são mais frequentes em áreas de maior vulnerabilidade social e menor capital social. Houve significativo incremento do quantitativo de médicos no Espírito Santo; entretanto, o provimento de médicos para a população em extrema pobreza não exibiu um indicador desejável, apresentando uma média ainda inaceitável em municípios de até 10.000 habitantes e aceitável nos municípios com as demais faixas populacionais.

Medicina de Família e Comunidade; Sistema Único de Saúde; Atenção

Primária à Saúde; Políticas Públicas de Saúde; Avaliação em Saúde

\author{
Correspondência \\ T. B. Emerich \\ Programa de Pós-graduação em Saúde Coletiva, Universidade \\ Federal do Espírito Santo. \\ Av. Marechal Campos 1468, Vitória, ES 29047-105, Brasil. \\ tatiemerich@hotmail.com \\ 1 Programa de Pós-graduação em Saúde Coletiva, Universidade \\ Federal do Espírito Santo, Vitória, Brasil. \\ 2 Escola de Governo, Fundação Oswaldo Cruz, Brasília, Brasil. \\ 3 University of Massachusetts Boston, Boston, U.S.A.
}




\section{Introdução}

O Sistema Único de Saúde (SUS) tem sido marcado por inúmeros desafios desde a sua criação há 30 anos. Apesar dos avanços obtidos, permanece o subfinanciamento, a necessidade de ampliação e resolutividade da atenção básica, a sobrecarga de doenças infecciosas e crônicas, as interferências do setor privado 1 , bem como a necessidade de fixação de profissionais comprometidos com o sistema 2,3,4. Essas lacunas afetam especialmente a população mais vulnerável e de extrema pobreza do país. Estimativas recentes apontam que a Região Sudeste é a que apresenta a menor taxa de pobreza $(17,6 \%)$ 5. Não obstante, alguns estados do Sudeste ainda exibem percentuais elevados de população em extrema pobreza. No ano de 2016, com base nos dados do CadÚnico, observa-se que o Espírito Santo exibiu uma taxa de $41,6 \%$ de pessoas em extrema pobreza 6 .

Nesse panorama, algumas iniciativas têm sido adotadas. Os governos petistas, especialmente o do ex-presidente Luiz Inácio Lula da Silva, foram marcados pelos programas de proteção social, com a priorização de uma agenda social que incluía políticas de combate à pobreza, como o Programa Bolsa Família ${ }^{7}$. Compromissos recentes também têm sido firmados a fim de cumprir os Objetivos do Desenvolvimento Sustentável proposto pela Organização das Nações Unidas (ONU) na Agenda 2030 e adotados pelo Brasil em 2015 8. Além disso, visando a modificar a situação de vulnerabilidade, especialmente desse contingente populacional, e garantir o acesso e a fixação de médicos em áreas prioritárias do país, uma das políticas adotadas na área da saúde foi o Programa Mais Médicos (PMM) 9. Esse Programa é uma política voltada para essa população mais vulnerável, a fim de diminuir as iniquidades em saúde 10, que fortalece a Estratégia Saúde da Família (ESF) 11, vai ao encontro de um dos objetivos do desenvolvimento sustentável 12, considera a escassez e os desequilíbrios na distribuição dos médicos um problema social e político, e se destaca como sendo a iniciativa mais efetiva devido à transversalidade de suas ações 3 .

É um Programa que teve grande repercussão midiática 13,14 e nas instituições médicas que, em sua maioria, foram contrárias à política 15,16 , principalmente pela inclusão de um acordo de cooperação entre o governo brasileiro e a Organização Pan-Americana da Saúde (OPAS) prevendo a vinda de médicos cubanos. Apesar dessa resistência, estudos têm demonstrado a grande aceitação por parte da população 17,18,19,20, dos gestores de saúde 17,18,19,21 e de outros profissionais da saúde 22 com o trabalho de médicos participantes do Programa (sejam eles brasileiros, intercambistas individuais ou cooperados), bem como os impactos positivos na saúde da população 23,24,25,26. Assim, diversos estudos nacionais e estaduais têm sido conduzidos para avaliar esse Programa.

A avaliação de programas é uma ferramenta preponderante para discussão, melhorias e recomendações sobre políticas públicas 27,28. Constitui uma importante ferramenta e pode ter diferentes focos, sejam eles na implantação, nos resultados e/ou nos impactos de uma política. Por ser um Programa recente, acredita-se que as avaliações de implantação do PMM são as mais oportunas.

O panorama do PMM no Espírito Santo, ainda foi pouco explorado pela literatura 29. Entretanto, a realidade capixaba destaca-se pela ambiguidade da coexistência de realidades distintas: alta concentração de médicos na capital Vitória (12,27 médicos por 1.000 habitantes) - maior razão entre as capitais do país - e pequena proporção de médicos no interior do estado (1,43 médico por 1.000 habitantes) 4 . Dessa maneira, este trabalho contribui para a literatura existente sobre o PMM ao criar um modelo lógico e indicadores inovadores que permitem avaliar a implantação do Programa em outros estados brasileiros, além da cooperação técnica Sul-Sul na área da saúde. Assim, este artigo teve por objetivo avaliar a implantação do PMM com base no estudo de caso do Espírito Santo.

\section{Métodos}

Trata-se de um estudo de avaliação da implantação do eixo provimento emergencial denominado Projeto Mais Médicos para o Brasil (PMMB) no PMM. Foi considerado o provimento médico de todos os 78 municípios que compõem o Estado do Espírito Santo. O trabalho contemplou a análise documental das leis que fundamentaram e instituíram o PMM, e tomando como base o modelo proposto, teve como objeto de análise dos dados o terceiro eixo do Programa, a saber: o PMMB que previa a inserção de médicos em municípios e unidades de saúde de regiões prioritárias do SUS, deficitárias 
destes profissionais de saúde. Assim, o estudo foi focado principalmente na análise da distribuição dos médicos nessas regiões.

O estudo foi dividido em duas etapas principais: (1) primeira - a análise documental das leis que instituíram o PMM; e (2) segunda - análise da implantação do PMMB no Espírito Santo.

Na primeira etapa foram analisadas a Portaria Interministerial no 1.369, de 8 de julho de 2013; a Portaria Normativa no 17, de 31 de julho de 2013 e a Lei no 12.871, de 22 de outubro de 2013, as quais propiciaram a construção de um modelo lógico do PMM.

A elaboração do modelo lógico de uma política e/ou programa constitui uma das prioridades na análise de uma intervenção. O modelo funciona como uma ferramenta de comunicação, de representação do funcionamento de um programa e permite o melhor entendimento dos projetos de ação, do processo de interação entre as ações, antecipando necessidades e consequências. Além disso, se expressa como uma projeção de como o programa deve funcionar e como pode ser constituído na prática 27 . Esse modelo consiste no primeiro passo de uma pesquisa avaliativa 27 e numa importante ferramenta para o planejamento e a análise dos dados desta pesquisa e de pesquisas futuras sobre a temática. Logo, a criação de um modelo lógico do PMM é pertinente.

Na segunda etapa, foi realizada uma análise de dados do PMMB no Espírito Santo por meio de pesquisas/coleta de informações na Secretaria de Estado da Saúde do Estado (SESA), bem como buscas nos bancos de dados do Cadastro Nacional de Estabelecimentos de Saúde (CNES), pertencentes ao Departamento de Informática do SUS (DATASUS), para a coleta de dados do número de médicos; no banco de dados do Instituto Brasileiro de Geografia e Estatística (IBGE); no DATASUS para a coleta da população residente estimada em 2012 e 2016; e no banco de dados do Instituto Jones dos Santos Neves (IJSN) para a verificação da população em extrema pobreza no ano de 2016.

Os dados foram analisados com base no porte populacional dos municípios que compõem o Espírito Santo por meio das seguintes faixas populacionais: até 10.000 habitantes; de 10.001 a 20.000; de 20.001 a 50.000; de 50.001 a 200.000; 200.001 habitantes ou mais, que tiveram como referência a divisão de porte populacional utilizada pelo IBGE, modificadas de acordo com a realidade do Espírito Santo.

A coleta de dados no DATASUS ocorreu pela pesquisa na Rede Assistencial, CNES - Recursos Humanos, a partir de agosto de 2007 - Ocupações classificadas pela CBO 2002 (Classificação Brasileira de Ocupações), selecionando os profissionais do Espírito Santo, tendo como referência os meses de julho de 2012 e julho de 2016, incluindo número de médicos totais e apenas da ESF.

As variáveis usadas nessa análise incluíram dados referentes ao total de médicos inseridos no provimento emergencial nos anos de 2013, 2014, 2015 e 2016 (1 triênio do programa) e o total de médicos desligados nesse período (2013 a 2016), que compuseram o perfil da implantação do PMM no Espírito Santo.

Tomou-se como referência dois dos quatro critérios prioritários de alocação de médicos do programa: "ter o Município 20\% (vinte por cento) ou mais da população vivendo em extrema pobreza, com base nos dados do Ministério do Desenvolvimento Social e Combate à Fome (MDS) (...) ou estar nas áreas referentes aos 40\% (quarenta por cento) dos setores censitários com os maiores percentuais de população em extrema pobreza dos Municípios" 30. Assim, partindo desses critérios e do pressuposto de que a população em extrema pobreza encontrava-se precariamente atendida pela assistência médica até a criação do Programa, propusemos a criação de um indicador que evidenciasse o número de médicos do PMM inseridos no SUS em relação à população estimada em extrema pobreza de acordo com a faixa populacional. Assim, temos:

$$
\text { Indice méd PMM pop ext pob }=\frac{\text { Total de médicos do provimento emergencial no ano de } 2016}{\text { População estimada de extrema pobreza do referido ano }} \times 1.000
$$

Adotamos como indicador ideal a proporção de 2,7 médicos para cada mil habitantes em situação de extrema pobreza. Essa proporção baseia-se no pressuposto da própria criação do PMM, uma vez que sua proposta é alcançar essa proporção de médicos no Brasil, semelhantemente ao que existe no Reino Unido, considerado um país com um dos melhores sistemas de saúde pública centrado na atenção básica 2,31. Criamos três categorias de provimento (inaceitável, aceitável e desejável) fundamentadas no número de médicos por 1.000 habitantes: 
(a) 0 a $0,99(<1)$ : provimento inaceitável;

(b) 1,0 a 1,99: provimento aceitável;

(c) 2,0 ou mais (>2): provimento desejável.

Os dados foram analisados no programa estatístico SPSS (https://www.ibm.com/). Foram realizados os testes não paramétricos de Wilcoxon e Kruskal-Wallis, uma vez que o número e o perfil de municípios nas faixas agrupadas são heterogêneos. Os cruzamentos resultaram do número de médicos dividido pela população residente no mesmo ano e multiplicado por uma base de 1.000 habitantes, a saber:

(a) Indicador médicos total ano 2012 = número de médicos do total ano 2012/população residente total ano 2012 x 1.000 habitantes;

(b) Indicador médicos ESF ano 2012 = número de médicos do ESF ano 2012/população residente total ano $2012 \times 1.000$ habitantes;

(c) Indicador médicos total ano 2016 = número de médicos do total ano 2016/população residente total ano $2016 \times 1.000$ habitantes;

(d) Indicador médicos ESF ano 2016 = número de médicos do ESF ano 2016/população residente total ano 2016 x 1.000 habitantes;

(e) Indicador médicos PMM para população extrema pobreza ano 2016 = total de médicos do provimento emergencial ano 2016/população total em extrema pobreza ano 2016 x 1.000 habitantes.

Após a realização desses cruzamentos, fez-se a classificação do provimento emergencial de acordo com o indicador proposto, o que permitiu delimitar a cobertura médica assistencial e avaliar a implantação do PMM no estado.

A pesquisa foi aprovada no Comitê de Ética em Pesquisa da Universidade Federal do Espírito Santo (CAAE 63949516.7.0000.5060).

\section{Resultados}

A construção do modelo lógico do PMM (Quadro 1) teve como pressuposto as perguntas: "Quem fazo quê??, "Quais são os recursos investidos?” e “Quais são as atividades previstas?” 27 (p. 69), bem como a representação esquemática proposta por Oliveira et al. 32 que inclui as dimensões, insumos, atividades, produtos, resultados e impacto. Trata-se de uma representação esquemática da lei que dispõe sobre o eixo do provimento emergencial que auxilia na análise documental do PMMB, sem considerar a plausibilidade lógica entre os componentes elencados pela política.

O Espírito Santo situa-se na Região Sudeste do país e conta com uma população estimada pelo IBGE para 2016 de 3.973.697 habitantes. Tem 78 municípios que compõem as quatro regiões de saúde: Norte, Central, Metropolitana e Sul. O estado tem um Índice de Desenvolvimento Humano (IDH) de 0,74 com grandes variações entre os municípios, de acordo com o perfil de cada um, desde municípios com IDH de 0,62 até municípios como a capital Vitória que tem o maior IDH do estado $(0,84)$. De modo semelhante, tem grandes desigualdades sociais, inclusive municípios com consideráveis contingentes populacionais vivendo em extrema pobreza (18,08\% em Brejetuba). Em consonância às desigualdades sociais, conta com uma população de 79,07\% coberta pela atenção básica, com variações entre os municípios. O Espírito Santo tinha no final do primeiro triênio do PMM (2016) 432 médicos integrantes do provimento emergencial, distribuídos nos 59 municípios $(75,64 \%)$ aderidos ao Programa.

Em relação ao perfil de implantação do PMM no Espírito Santo, observa-se na Tabela 1 que 38,5\% dos municípios estão na faixa populacional de 10.001 a 20.000 habitantes; 34,6\% de 20.001 a 50.000; $11,5 \%$ de até 10.000 habitantes; $9 \%$ de 50.001 a 200.000; e 6,4\% de 200.001 habitantes ou mais. Apesar do porte populacional de 200.001 habitantes ou mais representar o menor percentual de municípios, incluindo a capital do estado e quatro dos sete municípios da Região Metropolitana da Grande Vitória, estes são os que têm o maior percentual de população vivendo em extrema pobreza no estado (42,5\%), seguidos dos municípios de 20.001 a 50.000 habitantes (24\%) e de 50.001 a 200.000 (16,6\%).

Os municípios de 20.001 a 50.000 habitantes têm uma economia predominantemente agrícola e são em sua maioria de imigração italiana, pomerana e alemã. Já os municípios de 50.001 a 200.000 


\section{Quadro 1}

Modelo lógico das ações prioritárias do Programa Mais Médicos previstas na Lei no 12.871, de 22 de outubro de 2013. Brasil, 2013.

\begin{tabular}{|c|c|c|c|c|c|}
\hline DIMENSÕES & INSUMOS/RECURSOS & ATIVIDADES & PRODUTOS & $\begin{array}{c}\text { RESULTADOS } \\
\text { (IMEDIATOS E } \\
\text { INTERMEDIÁRIOS) }\end{array}$ & IMPACTO \\
\hline $\begin{array}{l}\text { Reordenação da } \\
\text { oferta de cursos } \\
\text { de Medicina } \\
\text { e Residência } \\
\text { Médica }\end{array}$ & \begin{tabular}{|c|} 
Chamamento público do \\
Ministério da Educação: \\
- Pré-seleção dos municípios; \\
- Critérios para autorização; \\
- Existência de redes \\
de atenção para o SUS: \\
atenção básica; urgência \\
e emergência; atenção \\
psicossocial; ambulatorial \\
especializada e hospitalar; \\
vigilância em saúde; critérios \\
de qualidade; \\
- Necessidade social \\
do curso para cidade e \\
região demonstrada por \\
indicadores
\end{tabular} & $\begin{array}{l}\text { Autorizar o } \\
\text { funcionamento de } \\
\text { cursos de Medicina } \\
\text { por instituições } \\
\text { privadas }\end{array}$ & $\begin{array}{l}\text { Abertura de novos } \\
\text { cursos de graduação } \\
\text { em Medicina em } \\
\text { instituições privadas }\end{array}$ & $\begin{array}{c}\text { Aperfeiçoamento de } \\
\text { médicos para atuação } \\
\text { nas políticas públicas de } \\
\text { saúde e na organização e } \\
\text { funcionamento do SUS }\end{array}$ & $\begin{array}{l}\text { Aprimoramento da } \\
\text { formação médica para } \\
\text { o SUS }\end{array}$ \\
\hline \multirow[t]{3}{*}{$\begin{array}{l}\text { Estabelecimento } \\
\text { de novos } \\
\text { parâmetros } \\
\text { para a formação } \\
\text { médica no país }\end{array}$} & $\begin{array}{c}\text { Oferta de vagas de } \\
\text { Programas de Residência } \\
\text { Médica: } \\
\text { - Medicina Geral de Família } \\
\text { e Comunidade (duração } \\
\text { mínima de } 2 \text { anos); } \\
\text { - Residência Médica de } \\
\text { acesso direto }\end{array}$ & \multirow[t]{3}{*}{\begin{tabular}{|c|} 
Implantar novas \\
diretrizes curriculares \\
para a formação \\
médica no Brasil
\end{tabular}} & \multirow{3}{*}{\begin{tabular}{|c} 
Novas diretrizes \\
curriculares \\
implantadas na \\
formação médica \\
com matriz curricular \\
dos cursos de \\
Medicina e requisitos \\
para a entrada \\
em Programa de \\
Residência Médica
\end{tabular}} & $\begin{array}{l}\text { Ampliação de vagas } \\
\text { de Residência Médica } \\
\text { no país }\end{array}$ & \multirow[t]{3}{*}{$\begin{array}{c}\text { Diminuição da } \\
\text { carência de médicos } \\
\text { para o SUS }\end{array}$} \\
\hline & $\begin{array}{c}\text { Carga horária mínima de } \\
\text { 30\% internato médico na } \\
\text { atenção básica e em serviços } \\
\text { de urgência e emergência } \\
\text { no SUS }\end{array}$ & & & $\begin{array}{l}\text { Formação médica } \\
\text { preparada e direcionada } \\
\text { para atuação no SUS }\end{array}$ & \\
\hline & \begin{tabular}{|c|} 
Oferta de vagas de \\
Residência Médica \\
equivalentes ao número \\
de egressos do curso de \\
Medicina no ano anterior \\
Obrigatoriedade do 1o ano \\
do Programa de Residência \\
em Medicina Geral de \\
Família e de Comunidade \\
para Programa de Residência \\
Médica em Medicina Interna \\
(Clínica Médica); Pediatria; \\
Ginecologia e Obstetrícia; \\
Cirurgia Geral; Psiquiatria; \\
Medicina Preventiva Social
\end{tabular} & & & $\begin{array}{c}\text { Aumento do número de } \\
\text { especialistas em Medicina } \\
\text { Geral de Família e } \\
\text { Comunidade }\end{array}$ & \\
\hline
\end{tabular}

(continua) 
Quadro 1 (continuação)

\begin{tabular}{|c|c|c|c|c|c|}
\hline DIMENSÕES & INSUMOS/RECURSOS & ATIVIDADES & PRODUTOS & $\begin{array}{l}\text { RESULTADOS } \\
\text { (IMEDIATOS E } \\
\text { INTERMEDIÁRIOS) }\end{array}$ & IMPACTO \\
\hline $\begin{array}{l}\text { Integração } \\
\text { ensino/serviço } \\
\text { para a promoção } \\
\text { em áreas } \\
\text { prioritárias } \\
\text { do SUS, de } \\
\text { aperfeiçoamento } \\
\text { de médicos para } \\
\text { a atenção básica }\end{array}$ & $\begin{array}{l}\text { Edital de preenchimento de } \\
\qquad \text { vagas: } \\
\text { I - Médico-participante; } \\
\text { II - Supervisor: médico } \\
\text { responsável pela supervisão } \\
\text { profissional do médico; } \\
\text { III - Tutor acadêmico: } \\
\text { docente médico responsável } \\
\text { pela orientação acadêmica. }\end{array}$ & $\begin{array}{l}\text { Seleção e ocupação } \\
\text { de vagas ofertadas. }\end{array}$ & $\begin{array}{l}\text { Distribuição de } \\
\text { médicos para atuar } \\
\text { em Unidades de } \\
\text { Saúde em áreas } \\
\text { prioritárias do SUS }\end{array}$ & $\begin{array}{l}\text { Inserção de médicos em } \\
\text { municípios/Unidades } \\
\text { de saúde de regiões } \\
\text { prioritárias }\end{array}$ & $\begin{array}{c}\text { Integração ensino/ } \\
\text { Serviço }\end{array}$ \\
\hline \multirow[t]{5}{*}{$\begin{array}{l}\text { PROJETO MAIS } \\
\text { MÉDICOS } \\
\text { PARA O BRASIL } \\
\text { (PMMB) }\end{array}$} & $\begin{array}{c}\text { Atuação de uma instituição } \\
\text { pública de Educação } \\
\text { Superior }\end{array}$ & \begin{tabular}{|c|} 
Aperfeiçoar a \\
formação dos \\
médicos participantes \\
do PMMB \\
Realizar módulo \\
de acolhimento (4 \\
semanas). \\
Conteúdo: SUS - \\
funcionamento, \\
protocolos, língua \\
portuguesa, Código \\
de Ética Médica
\end{tabular} & $\begin{array}{c}\text { Médicos capacitados } \\
\text { para atuarem em } \\
\text { unidades básicas do } \\
\text { SUS }\end{array}$ & & \\
\hline & Recursos da União & $\begin{array}{c}\text { Custear despesas } \\
\text { de deslocamento } \\
\text { dos médicos } \\
\text { participantes e seus } \\
\text { dependentes legais } \\
\text { Pagamento de: } \\
\text { I - Bolsa-formação } \\
\text { II - Bolsa-supervisão } \\
\text { III - Bolsa-tutoria }\end{array}$ & $\begin{array}{l}\text { Recursos da União } \\
\text { sendo gastos para } \\
\text { custear o PMMB }\end{array}$ & & \\
\hline & \multirow[t]{2}{*}{$\begin{array}{c}\text { Atuação do Ministério da } \\
\text { Educação e Ministério da } \\
\text { Saúde }\end{array}$} & $\begin{array}{l}\text { Emitir número } \\
\text { de registro único } \\
\text { para os médicos } \\
\text { intercambistas } \\
\text { e comunicar ao } \\
\text { Conselho de } \\
\text { Medicina }\end{array}$ & $\begin{array}{l}\text { Registros únicos } \\
\text { emitidos aos } \\
\text { intercambistas } \\
\text { cooperados }\end{array}$ & & \\
\hline & & Coordenar o PMMB & $\begin{array}{c}\text { Definição da } \\
\text { coordenação do } \\
\text { PMMB no estado }\end{array}$ & & \\
\hline & Atuação dos municípios & $\begin{array}{l}\text { Inscrever nos editais } \\
\text { e contrapartidas } \\
\text { (inserir médicos nas } \\
\text { equipes; oferecer } \\
\text { moradia; garantir } \\
\text { alimentação; não } \\
\text { substituir médicos } \\
\text { que já componham } \\
\text { equipes) }\end{array}$ & $\begin{array}{c}\text { Assinatura do } \\
\text { termo de adesão } \\
\text { e compromisso do } \\
\text { município }\end{array}$ & & \\
\hline
\end{tabular}


Caracterização da implantação do Programa Mais Médicos no Espírito Santo, Brasil, de acordo com o perfil de médicos atuantes, desligados e a faixa populacional, 2016.

\begin{tabular}{|c|c|c|c|c|c|c|c|c|c|c|c|c|}
\hline & \multicolumn{10}{|c|}{ Faixa populacional (habitantes) } & \multicolumn{2}{|c|}{ Total } \\
\hline & \multicolumn{2}{|c|}{ Até 10.000} & \multicolumn{2}{|c|}{$\begin{array}{l}10.001 \mathrm{a} \\
20.000\end{array}$} & \multicolumn{2}{|c|}{$\begin{array}{c}20.001 a \\
50.000\end{array}$} & \multicolumn{2}{|c|}{$\begin{array}{l}50.001 \text { a } \\
200.000\end{array}$} & \multicolumn{2}{|c|}{$\begin{array}{c}200.001 \text { ou } \\
\text { mais }\end{array}$} & \multirow[b]{2}{*}{$\mathbf{n}$} & \multirow[b]{2}{*}{$\%$} \\
\hline & $\mathbf{n}$ & $\%$ & $\mathbf{n}$ & $\%$ & $\mathbf{n}$ & $\%$ & $\mathbf{n}$ & $\%$ & $\mathbf{n}$ & $\%$ & & \\
\hline \multicolumn{13}{|l|}{ Municípios } \\
\hline Número de municípios & 9 & 11,5 & 30 & 38,5 & 27 & 34,6 & 7 & 9,0 & 5 & 6,4 & 78 & 100,0 \\
\hline População geral & 68.713 & 1,73 & 410.095 & 10,32 & 805.538 & 20,27 & 761.077 & 19,15 & 1.928 .274 & 48,53 & 3.973 .697 & 100,0 \\
\hline População em extrema pobreza & 15.836 & 3,4 & 62.653 & 13,5 & 111.751 & 24,0 & 77.454 & 16,6 & 198.020 & 42,5 & 465.714 & 100,0 \\
\hline \multicolumn{13}{|l|}{ Total médicos do provimento emergencial } \\
\hline CRM Brasil & 0 & 0,0 & 10 & 7,4 & 25 & 18,5 & 34 & 25,1 & 66 & 48,8 & 135 & 100,0 \\
\hline $\begin{array}{l}\text { Intercambistas individuais (brasileiros e } \\
\text { estrangeiros) }\end{array}$ & 0 & 0,0 & 0 & 0,0 & 2 & 9,5 & 13 & 61,9 & 6 & 28,5 & 21 & 100,0 \\
\hline Intercambistas cooperados (cubanos) & 3 & 1,0 & 27 & 9,7 & 67 & 24,2 & 75 & 27,1 & 104 & 37,6 & 276 & 100,0 \\
\hline \multicolumn{13}{|l|}{ Total médicos desligados } \\
\hline CRM Brasil & 1 & 1,2 & 8 & 9,4 & 13 & 15,3 & 15 & 17,6 & 48 & 56,5 & 85 & 100,0 \\
\hline $\begin{array}{l}\text { Intercambistas individuais (brasileiros e } \\
\text { estrangeiros) }\end{array}$ & 0 & 0,0 & 0 & 0,0 & 0 & 0,0 & 0 & 0,0 & 2 & 100 & 2 & 100,0 \\
\hline Intercambistas cooperados (cubanos) & 1 & 0,8 & 12 & 10,1 & 17 & 14,4 & 24 & 20,3 & 65 & 55,0 & 118 & 100,0 \\
\hline
\end{tabular}

CRM Brasil: médicos brasileiros com registro no Conselho Regional de Medicina do Espírito Santo.

Fonte: elaborada pelos autores.

habitantes são economicamente mais fortes e com maior infraestrutura devido à proximidade da Região Metropolitana da Grande Vitória.

Em relação ao total de médicos do provimento emergencial para o Espírito Santo (432), verificouse que $63,8 \%$ pertencem ao perfil intercambistas cooperados (cubanos) e 31,2\% a médicos brasileiros com registro no Conselho Regional de Medicina (CRM). Sobre esse total em relação ao perfil municipal, 48,8\% dos médicos "CRM Brasil” foram alocados em municípios de 200.001 habitantes ou mais; $25,1 \%$ de 50.001 a 200.000 habitantes; e $18,5 \%$ de 20.001 a 50.000. Os intercambistas individuais estavam predominantemente nos municípios de 50.001 a 200.00 habitantes (61,9\%); e os intercambistas cooperados se distribuíam de forma mais heterogênea: 37,6\% alocados em municípios de 200.001 habitantes ou mais; 27,1\% em municípios de 50.001 a 200.000; e 24,2\% de 20.001 a 50.000 habitantes.

Sobre o perfil de desligamento dos médicos participantes, do total de médicos desligados 57,5\% foram do perfil intercambistas cooperados; 41,4\% do perfil "CRM Brasil" e apenas 0,8\% do perfil intercambistas individuais.

A Tabela 2 evidencia que em todas as faixas populacionais a média aumentou de 2012 para 2016, tanto no indicador de médicos da ESF quanto no indicador de médicos totais do sistema de saúde brasileiro. Para o Espírito Santo como um todo, o teste de Wilcoxon mostra que o aumento no indicador de médicos da ESF e de médicos totais, de 2012 para 2016, foi significativo, com valor de $\mathrm{p}=0,005$ e valor de $\mathrm{p}<0,001$, respectivamente.

Em relação ao número de médicos da ESF, observa-se que no ano de 2012 este indicador não foi significativo $(\mathrm{p}=0,329)$, revelando não haver diferença entre as faixas de municípios. Já no ano de 2016, esse indicador foi significativo ( $p=0,012$ ), evidenciando diferenças entre as faixas populacionais, possivelmente devido ao maior aumento no indicador para os municípios menores (de até 20.000 habitantes). Observa-se ainda que houve um aumento na média dos indicadores de médicos da ESF do ano de 2012 para o de 2016 em todas as faixas municipais, mesmo este indicador se mantendo inaceitável $(0$ a 0,99) para todos os grupos de municípios em 2016. 
Tabela 2

Indicadores do número de médicos da Estratégia Saúde da Família (ESF), total e a proposta do indicador de provimento de médicos para a população em extrema pobreza. Espírito Santo, Brasil, 2016.

\begin{tabular}{|c|c|c|c|c|c|c|c|c|c|c|}
\hline \multirow{2}{*}{$\begin{array}{l}\text { Faixa populacional de } \\
\text { 2016/Estatística }\end{array}$} & \multicolumn{4}{|c|}{ Indicadores antes (2012) } & \multicolumn{4}{|c|}{ Indicadores depois (2016) } & \multicolumn{2}{|c|}{ Provimento * } \\
\hline & ESF & $\begin{array}{l}\text { Valor } \\
\text { de } p * *\end{array}$ & Total & $\begin{array}{c}\text { Valor } \\
\text { de } p * *\end{array}$ & ESF & $\begin{array}{l}\text { Valor de } \\
\qquad p * *\end{array}$ & Total & $\begin{array}{c}\text { Valor } \\
\text { de } p * *\end{array}$ & PMM 2016 & $\begin{array}{c}\text { Valor } \\
\text { de } p * *\end{array}$ \\
\hline Até 10.000 & & 0,329 & & 0,002 & & 0,012 & & 0,001 & & 0,001 \\
\hline Média & 0,15 & & 0,70 & & 0,23 & & 0,68 & & 0,13 & \\
\hline Número de médicos & 9 & & 9 & & 9 & & 9 & & 9 & \\
\hline Desvio padrão & 0,13 & & 0,33 & & 0,07 & & 0,43 & & 0,20 & \\
\hline \multicolumn{11}{|l|}{10.001 a 20.000} \\
\hline Média & 0,11 & & 0,83 & & 0,23 & & 0,91 & & 1,00 & \\
\hline Número de médicos & 30 & & 30 & & 30 & & 30 & & 30 & \\
\hline Desvio padrão & 0,08 & & 0,77 & & 0,10 & & 0,68 & & 2,06 & \\
\hline \multicolumn{11}{|l|}{20.001 a 50.000} \\
\hline Média & 0,13 & & 0,94 & & 0,17 & & 1,08 & & 1,06 & \\
\hline Número de médicos & 27 & & 27 & & 27 & & 27 & & 27 & \\
\hline Desvio padrão & 0,09 & & 0,52 & & 0,09 & & 0,62 & & 1,03 & \\
\hline \multicolumn{11}{|l|}{50.001 a 200.00} \\
\hline Média & 0,07 & & 1,29 & & 0,19 & & 1,51 & & 1,88 & \\
\hline Número de médicos & 7 & & 7 & & 7 & & 7 & & 7 & \\
\hline Desvio padrão & 0,05 & & 0,78 & & 0,07 & & 0,81 & & 0,67 & \\
\hline \multicolumn{11}{|l|}{200.001 ou mais } \\
\hline Média & 0,06 & & 2,71 & & 0,09 & & 2,85 & & 1,03 & \\
\hline Número de médicos & 5 & & 5 & & 5 & & 5 & & 5 & \\
\hline Desvio padrão & 0,05 & & 1,55 & & 0,04 & & 1,38 & & 0,57 & \\
\hline \multicolumn{11}{|l|}{ Espírito Santo } \\
\hline Média & $0,11 * \star \star$ & & $1,01 \#$ & & $0,19 * * *$ & & $1,12 \#$ & & 1,00 & \\
\hline Número de médicos & 78 & & 78 & & 78 & & 78 & & 78 & \\
\hline Desvio padrão & 0,08 & & 0,85 & & 0,09 & & 0,85 & & 1,47 & \\
\hline
\end{tabular}

Fonte: elaborada pelos autores.

* População em extrema pobreza;

** Teste de Kruskal-Wallis:

*** Teste Wilcoxon ESF 2012 x ESF 2016; valor de $\mathrm{p}=$ 0,005;

\# Teste Wilcoxon Total 2012 x Total 2016; valor de $p<0,001$.

Média inaceitável $(0$ a 0,99)

Média aceitável (1,0 a 1,99)

Média desejável (2,0 ou mais)

Em relação ao número de médicos totais, tanto em 2012 quanto em 2016, esse indicador foi significativo com $\mathrm{p}=0,002$ (ano de 2012) e $\mathrm{p}=0,001$ (ano de 2016), substanciando diferenças entre as faixas de municípios. Os municípios de até 10.000 habitantes e os de 10.001 a 20.000 habitantes mantiveram-se com um padrão considerado inaceitável na média do indicador do número de médicos. Os municípios de 20.001 a 50.000 habitantes mudaram de um indicador inaceitável em 2012 (média = 0,94) para um indicador aceitável em 2016 (média = 1,08). Já os municípios de 50.001 a 200.000 habitantes permaneceram com um indicador aceitável de 2012 a $2016(1,0$ a 1,99) e os municípios 200.001 habitantes ou mais continuaram com uma média do indicador de médicos totais considerada desejável (2,0 ou mais médicos/1.000 habitantes).

Analisando a Tabela 2 em relação às faixas populacionais, observa-se que comparando os médicos atuantes na ESF no ano de 2012 tendo como critério o porte populacional, não se evidencia grandes disparidades entre os indicadores, uma vez que este indicador foi baixo tanto nos municípios de até 
10.000 habitantes $(0,15)$ quanto nos municípios com 200.001 ou mais $(0,06)$. No entanto, no ano de 2012, ao se comparar o indicador de médicos nos municípios de até 10.000 habitantes com os municípios de grande porte (200.001 habitantes ou mais), fica clara a notável desigualdade entre esses indicadores, 0,7 e 2,71, respectivamente. Apesar da incorporação do provimento médico com o Programa no Espírito Santo, essa desigualdade persistiu porque a proporção de médicos nos municípios de 200.001 habitantes ou mais foi quatro vezes maior do que nos municípios de até 10.000 habitantes.

No que tange ao provimento de médicos em relação à população em extrema pobreza, a análise mostra que apenas os municípios de até 10.000 habitantes exibiram uma média inaceitável de médicos por 1.000 habitantes (média $=0,13$ ). Todos os demais grupos de municípios apresentaram um indicador aceitável de médicos, sendo a maior média encontrada para os municípios de 50.001 a 200.000 habitantes (média $=1,88$ ). Não obstante, nenhuma das faixas populacionais exibiu um indicador desejável do número de médicos do Programa em relação à população mais vulnerável, indicando uma implantação parcial do PMM, ainda insuficiente para cobrir as carências no provimento médico assistencial.

\section{Discussão}

O delineamento do modelo lógico do PMM proposto neste estudo evidencia, em primeiro lugar, a complexidade deste Programa e sua estruturação em três ações prioritárias: a reordenação da oferta de cursos de Medicina e Residência Médica; o estabelecimento de novos parâmetros para a formação médica no país; e a integração ensino/serviço para promoção em áreas prioritárias do SUS, de aperfeiçoamento de médicos para a atenção básica, por meio do PMMB. Comprova também os muitos insumos e recursos que esse Programa requer, bem como as distintas atividades que ele inclui. Como se trata de um programa que foi implantado de forma emergencial, esse modelo adquire relevância, já que seu funcionamento está descrito, por partes, em distintas leis, portarias ministeriais e termos de cooperação, fazendo com que seu entendimento se torne, por vezes, demasiadamente confuso e necessite da articulação de todos os documentos para a devida transparência da implantação e consequente monitoramento, avaliação e análises.

Vale ressaltar ainda que esse modelo não se constitui em uma representação única sobre esse Programa. Outros modelos podem ser propostos e pertinentes para analisá-lo em sua complexidade.

A proposta de um indicador de médicos participantes do PMM em relação à população em extrema pobreza vai ao encontro dos pressupostos da criação do Programa, já que a prioridade de alocação dos médicos ocorreu seguindo o princípio da equidade e considerando a vulnerabilidade populacional. Conforme já demonstrado no estudo de Pinto et al. ${ }^{19}$, a proporção brasileira de médicos alocados em municípios de extrema pobreza foi quase três vezes maior do que nas capitais e municípios mais ricos, semelhantemente ao que ocorre em outras regiões do país.

O perfil de municípios incluídos nas faixas populacionais do Estado do Espírito Santo também revela que é um estado com municípios majoritariamente de pequeno porte, com população de até 50.000 habitantes (73,1\%), o que é condizente com os objetivos do PMMB.

O perfil de médicos encontrado no presente trabalho de prioritariamente intercambistas cooperados $(63,8 \%)$ é condizente com o perfil verificado para o país, apesar de um pouco abaixo da média brasileira, conforme verificado para o Brasil nos anos de 2013 e 2014 por Pereira et al. 33 (79\%); por Oliveira 34 (78,69\%); e por Santos et al. 12 para os anos de 2013 a 2015 (72,5\%). Portanto, não é surpreendente que um grande percentual de médicos desligados no Espírito Santo corresponda a médicos cubanos (57,5\%). Sem embargo, o percentual de 41,4\% de médicos com "CRM Brasil" desligados nesse estado também chama a atenção e remonta à grande dificuldade de fixação de médicos brasileiros no SUS. O estudo de Araújo et al. 35 esclarece, em relação aos médicos concluintes do Programa Nacional de Valorização da Atenção Básica (PROVAB) que migraram para esse programa, que se tratavam principalmente de médicos jovens (faixa etária de 30 anos de idade), sem família construída, recém-formados, a maioria solteiros (69\%) e que não tinham feito nenhuma especialização anterior ao PROVAB; sua motivação principal era participar do PROVAB e do PMM para adquirirem a pontuação de $10 \%$ para as provas de residência médica. Segundo os dados analisados, a motivação de médicos brasileiros para atuação na atenção básica e no SUS se restringiu, pelo menos ao longo do primeiro 
triênio de implantação do PMM, a um caráter instrumental para facilitar a entrada em programas de residência médica, muitos deles focados em especializações que não incluem o exercício da medicina de família 36. Essa evasão traz uma grande desvantagem aos municípios, uma vez que em alguns deles observou-se demora na reposição do profissional por outro médico participante 36 .

Além disso, o maior percentual de médicos cubanos inseridos no programa confirma, de certa forma, a recusa de médicos brasileiros em participar do PMM nos três primeiros anos de implantação. Essa insatisfação da classe médica com o Programa pode ser vista nas repercussões midiáticas que incluíram como principais personagens os médicos cooperados 13, bem como na forte influência negativa e desestimuladora das instituições médicas na implantação do Programa no Mato Grosso 37.

O aumento significativo do indicador de médicos da ESF e de médicos totais, de 2012 para 2016, sugere que o PMM tem cumprido ou pelo menos tem contribuído para a diminuição da carência de médicos em áreas prioritárias. Essa hipótese é reforçada pelos dados do indicador de médicos do provimento emergencial em relação à população em extrema pobreza, que evidenciou que, à exceção dos municípios de até 10.000 habitantes, todos os demais grupos de municípios apresentaram um indicador aceitável de médicos após a incorporação do programa, com destaque para os municípios de 50.001 a 200.000 habitantes que exibiram um indicador de 1,88 médico do PMM para cada 1.000 habitantes em extrema pobreza. Outro estudo demonstra que o Programa contribuiu para um crescimento de aproximadamente $10,7 \%$ do número de médicos no país de 2012 a 2015. Caso não houvesse a implantação desse Programa, em quatro estados brasileiros não teria ocorrido nenhum aumento ou ocorreria até mesmo uma diminuição do número de médicos 19. Esse aumento na proporção de médicos tem favorecido o crescimento da cobertura populacional da atenção básica no país, que aumentou quatro vezes quando comparada com o crescimento médio esperado em anos anteriores à implantação do Programa 19.

Apesar do aumento verificado, o indicador referente ao número de médicos de família permaneceu inaceitável $(0$ a 0,99) em todos os grupos de municípios, mesmo após os três primeiros anos do PMM. Esses dados corroboram os encontrados por Miranda \& Melo 38 para o Brasil, em relação ao número de médicos com atuação no SUS e na atenção básica em dezembro de 2013.

A mudança mais contundente observada no presente trabalho ocorreu em relação ao número de médicos totais de 2012 para 2016, nos municípios de 20.001 a 50.000 habitantes, os quais saíram de um padrão considerado inaceitável para um padrão aceitável de médicos por 1.000 habitantes. Entretanto, comparando esses dados com a média nacional de médicos cadastrados nos serviços de saúde do país, observa-se que o indicador encontrado para o Espírito Santo, para essa faixa de municípios, mesmo com a incorporação do PMM, ainda está muito abaixo da média nacional, que foi de 2,5 médicos/1.000 habitantes na faixa de municípios de 25.000 a 49.999 habitantes em 201338 .

Para o período de 2013 a 2015, os dados de um estudo nacional também demonstram que o PMMB contribuiu para a diminuição da carência de médicos. Houve um aumento no número de municípios com a relação $\geq 1,0$ médico/1.000 habitante, que subiu de 163 para 348 municípios de 2013 para 2015. A cobertura da atenção primária também aumentou, passando de 77,9\% em 2013 para 86,3\% em 2015. Esse aumento refletiu na diminuição de 8,8\% das internações por condições sensíveis à atenção primária nos municípios inseridos do PMMB, taxa que permaneceu inalterada nos municípios não inscritos, comprovando o impacto positivo do programa 12 .

Se forem analisados os dados nacionais referentes à implantação do PMMB em ambientes rurais, remotos, socialmente vulneráveis e com limitado acesso aos serviços de saúde, nos anos de 2013 a 2014, observa-se que em todas as regiões do país houve um aumento percentual na proporção de médicos $\geq 1,0 / 1.000$ habitantes. De modo semelhante, houve uma diminuição percentual em todas as regiões na proporção de médicos $<0,1 / 1.000$ habitantes após o Programa, sendo esta diminuição mais expressiva na Região Nordeste, onde baixou de 17,2\% para 4,4\%. Na Região Sudeste, a proporção de médicos antes e depois do Programa passou de 13,4\% para um indicador de $\geq 0,7$ e 1,0 para 21,1\%. Não obstante, embora as dificuldades tenham diminuído, elas não foram eliminadas, haja vista, por exemplo, as proporções de médicos antes e depois do Programa (28,2\% antes e 28,9\% depois) para o indicador de $\geq 0,4$ e $<$ 0,7 médicos/1.000 habitantes na Região Sudeste 33 .

A disparidade no provimento médico entre municípios de pequeno porte comparados aos municípios de grande porte, mesmo após a implantação do PMMB, reforça a grande concentração de médicos em centros urbanos, à semelhança do perfil exibido pela distribuição de médicos no país 38 . 
Um dos fatores que contribuem para essas disparidades é o fato de os centros urbanos concentrarem as maiores oportunidades de trabalho, estudo, proximidade do lazer, maior PIB e IDH, menores níveis de vulnerabilidade social, melhores remunerações, planos de carreira, condições para exercer o trabalho e valorização profissional 3 .

Esses indicadores evidenciam que apesar dos inúmeros desafios enfrentados em sua implantação, esse Programa conseguiu alcançar os objetivos que se propôs. Dentre os obstáculos vivenciados pelo PMMB, estão: a falta de apoio das próprias universidades federais em se posicionarem favoráveis ao Programa; a falta de apoio de secretarias estaduais de saúde em priorizar o desenvolvimento e as articulações necessárias ao bom andamento do mesmo; a falta de articulação do Ministério da Saúde com as secretarias estaduais que coordenam o PMMB, já que não são chamadas para discutir as dificuldades e formas de melhorias; a falta de esclarecimento do Ministério da Saúde sobre algumas questões pertinentes ao Programa, como o desligamento ou remanejamento de médicos e solução de problemas éticos ${ }^{37}$. Além disso, uma recente publicação da OPAS aponta algumas críticas dos médicos cubanos ao PMM e incluem a falta de assistência aos médicos atuantes em áreas isoladas, a falta de parcerias a longo prazo com instituições de pesquisa e outras, a afim de garantir a continuidade das ações, a necessidade de inclusão de cursos preparatórios que incluam a história e culturas do Brasil, dentre outras 39 .

Não obstante os desafios, o PMMB tem sido apontado como um Programa que contribuiu no estímulo da educação permanente e organização do modelo de trabalho das unidades básicas de saúde, fortalecendo a atenção básica, estimulando a qualificação dos serviços de saúde e aumentando o acesso da população aos serviços de saúde 37 .

\section{Considerações finais}

O delineamento do modelo lógico do PMM orientou a análise da implantação do programa no Espírito Santo, principalmente no que tange a dimensão integração ensino-serviço para promoção de aperfeiçoamento de médicos para a atenção básica. Assim, o presente estudo revelou desde o cenário da distribuição das populações que vivem com menos de 140 reais por mês no Espírito Santo - consideradas em extrema pobreza - até o panorama da implantação desse programa com alguns resultados nessas realidades.

Resumidamente, quatro dos sete municípios da Região Metropolitana da Grande Vitória concentram o maior percentual de população vivendo em extrema pobreza no estado $(42,5 \%)$, seguidos dos municípios de 20.001 a 50.000 habitantes (24\%) e de 50.001 a 200.000 (16.6\%). Do total de médicos designados para o provimento emergencial para o Espírito Santo (432), a maioria $(63,8 \%)$ pertencia ao perfil intercambistas cooperados (cubanos) e 31,2\% a médicos com CRM brasileiro. Destaca-se que tanto os médicos brasileiros quanto os intercambistas individuais foram alocados prioritariamente em municípios de médio e grande portes, e os cubanos se distribuíram de forma mais heterogênea em municípios de todos os portes populacionais e, consequentemente, são mais frequentes em áreas de maior vulnerabilidade social e menor capital social.

Assim, percebeu-se que, apesar do significativo incremento do quantitativo de médicos no Espírito Santo de uma maneira geral em 2016 em relação ao ano de 2012, o provimento de médicos para a população em extrema pobreza no estado não exibiu um indicador desejável, apresentando uma média ainda inaceitável em municípios de até 10.000 habitantes e aceitável nos municípios com as demais faixas populacionais. Esses dados reforçam a necessidade de manutenção e ampliação desse Programa, que contribui de forma incontestável no fortalecimento da atenção básica com o aumento da cobertura do modelo de medicina familiar e a melhoria dos indicadores de saúde. Além disso, o Programa põe em evidência importantes lições relegadas pelas boas práticas e experiências dos profissionais cubanos, que incluem a mudança na relação médico/comunidade somadas à interação socioprofissional e sociocultural. 


\section{Colaboradores}

T. B. Emerich participou da coleta, análise e interpretação dos dados e redação do manuscrito. A. G. Cavaca, E. T. Santos Neto e A. E. Oliveira participaram da concepção, análise e interpretação dos dados e revisão crítica. C. E. Siqueira participou da interpretação dos dados e revisão crítica final do manuscrito.

\section{Informações adicionais}

ORCID: Tatiana Breder Emerich (0000-0003-41040562); Aline Guio Cavaca (0000-0001-7314-584X); Edson Theodoro dos Santos Neto (0000-00027351-7719); Adauto Emmerich Oliveira (00000002-9679-8592); Carlos Eduardo Siqueira (00000001-8993-3031).

\section{Referências}

1. Paim J, Travassos C, Almeida CM, Bahia L, Macinko J. O sistema de saúde brasileiro: história, avanços e desafios. Saúde no Brasil 1. Lancet 2011; 6736:11-31.

2. Garcia B, Rosa L, Tavares F. Projeto mais médicos para o Brasil: apresentação do programa e evidências acerca de seu sucesso. Informações FIPE 2014; (402):26-36.

3. Oliveira APC, Gabriel M, Dal Poz MR, Dussault G. Desafios para assegurar a disponibilidade e acessibilidade à assistência médica no Sistema Único de Saúde. Ciênc Saúde Colet 2017; 22:1165-80.

4. Scheffer M, organizador. Demografia médica no Brasil. São Paulo: Conselho Regional de Medicina do Estado de São Paulo/Faculdade de Medicina, Universidade de São Paulo; 2018.

5. Instituto Jones dos Santos Neves. Síntese dos Indicadores Sociais do Espírito Santo PNAD 2015. Vitória: Instituto Jones dos Santos Neves; 2016.

6. Instituto Jones dos Santos Neves. Perfil da pobreza no Espírito Santo: famílias inscritas no CadÚnico 2016. Vitória: Instituto Jones dos Santos Neves; 2017.

7. Machado CV, Lima LD, Baptista TWF, Castro ALB. Política de saúde no Governo Lula: continuidades e mudanças. Revista Política, Planejamento e Gestão em Saúde 2010; 1:11-32.

8. Departamento de Vigilância de Doenças e Agravos não Transmissíveis e Promoção da Saúde, Secretaria de Vigilância em Saúde, Ministério da Saúde. Saúde Brasil 2017: uma análise da situação de saúde e os desafios para o alcance dos objetivos de desenvolvimento sustentável. Brasília: Ministério da Saúde; 2018.

\section{Agradecimentos}

O presente trabalho foi realizado com o apoio da Coordenação de Aperfeiçoamento de Pessoal de Nível Superior (CAPES - código de financiamento 001).

9. Brasil. Lei no 12.871 , de 22 de outubro de 2013. Institui o Programa Mais Médicos, altera as Leis n. 8.745, de 9 de dezembro de 1993, e n. 6.932, de 7 de julho de 1981, e dá outras providências. Diário Oficial da União 2013; 23 out.

10. Santos LMP, Costa AM, Girardi SN. Programa Mais Médicos: uma ação efetiva para reduzir iniquidades em saúde. Ciênc Saúde Colet 2015; 20:3547-52.

11. Macinko J, Harris MJ. Brazil's family strategy - delivering community-based primary care in a universal health system. N Engl J Med 2015; 372:2177-81.

12. Santos LMP, Oliveira A, Trindade JS, Barreto ICHC, Palmeira PA, Comes Y, et al. Implementation research: towards universal health coverage with more doctors in Brazil. Bull World Health Organ 2017; 95:103-12.

13. Faria MA, Rocha CMF, Brites LS, Machado AMG, Santos CAR, Cechinel NFR, et al. Programa Mais Médicos e sua repercussão na mídia: o que informar e para quem? RECIIS (Online) 2018; 12:14-25.

14. Emerich TB, Oliveira AE, Cavaca AG, Santos-Neto ET, Malinverni C. Na saúde e na imprensa: análise midiática da implantação do Programa Mais Médicos. In: Organização Pan-Americana da Saúde, editor. Interações socioculturais dos médicos cubanos participantes do Programa Mais Médicos no Brasil. Brasília: Organização Pan-Americana da Saúde; 2018. p. 33-55. 
15. Jesus RA, Medina MG, Prado NMBL. Programa Mais Médicos: análise documental dos eventos críticos e posicionamentos dos atores sociais. Interface (Botucatu, Online) 2016; 21 Suppl 1:1241-55.

16. Lopes MACP, Costa MJM, Santana RP, Pinheiro CO. Percepção de usuários do Sistema Único de Saúde acerca do Programa Mais Médicos em um município do interior do Ceará. Rev Bras Pesqui Saúde 2017; 19:50-7.

17. Pereira LL, Silva HP, Santos LMP. Projeto Mais Médicos para o Brasil: estudo de caso em comunidades quilombolas. Rev ABPN 2015; 7:28-51.

18. Santos JBF, Maciel RHM, Maia ALLN, Guimarães EPA. Médicos estrangeiros no Brasil: a arte do saber olhar, escutar e tocar. Saúde Soc 2016; 25:1003-16.

19. Pinto H, Oliveira FP, Santana JSS, Santos FOS, Araújo SQ. Programa Mais Médicos: avaliando a implantação do eixo provimento de 2013 a 2015. Interface (Botucatu, Online) 2016; 21 Suppl 1:1087-101.

20. Galavote HC, Franco TB, Freitas PSS, Lima EFA, Garcia ACP, Andrade MAC, et al. A gestão do trabalho na estratégia saúde da família: (des)potencialidades no cotidiano do trabalho em saúde. Saúde Soc 2016; 25:988-1002.

21. Arruda CAM, Pessoa VM, Barreto ICHC, Carneiro FF, Comes Y. Percepções de gestores municipais de saúde sobre o provimento e a atuação dos médicos do Programa Mais Médicos. Interface (Botucatu, Online) 2016; 21 Suppl 1:1269-80.

22. Comes Y, Trindade JS, Pessoa VM, Barreto ICHC, Shimizu HE, Dewes D, et al. A implementação do Programa Mais Médicos e a integralidade nas práticas da Estratégia Saúde da família. Ciênc Saúde Colet 2016; 21:2729-38.

23. Gonçalves RF, Souza IMC, Tanaka OY, Santos CR, Brito-Silva K, Santos LX, et al. Programa Mais Médicos no Nordeste: avaliação das internações por condições sensíveis à Atenção Primária à Saúde. Ciênc Saúde Colet 2016; 21:2815-24.

24. Alencar APA, Xavier SPL, Laurentino PAS, Lira PF, Nascimento VB, Carneiro N, et al. Impacto do Programa Mais Médicos na atenção básica de um município do sertão central nordestino. Revista Eletrônica Gestão e Sociedade 2016; 10:1290-301.

25. Miranda GMD, Mendes ACG, Silva ALA, Santos Neto PM. A ampliação das equipes de saúde da família e o Programa Mais Médicos nos municípios brasileiros. Trab Educ Saúde 2017; 15:131-45

26. Mourão Netto JJ, Rodrigues ARM, Aragão OC, Goyanna NF, Cavalcante AES, Vasconcelos MAS, et al. Programa Mais Médicos e suas contribuições para a saúde no Brasil: revisão integrativa. Rev Panam Salud Pública 2018; 42:e2.

27. Brousselle A, Champagne F, Contandriopoulos A-P, Hartz Z. Avaliação: conceitos e métodos. Rio de Janeiro: Editora Fiocruz; 2011.
28. Vieira-da-Silva LM. Avaliação de políticas e programas de saúde. Rio de Janeiro: Editora Fiocruz; 2014.

29. Martins ACP, Sant'Anna PA, Rocha JFD, Vieira MA. Programa Mais Médicos: uma revisão integrativa. Rev Bras Promoç Saúde 2017; 30:1-14.

30. Ministério da Saúde; Ministério da Educação. Portaria Interministerial no 1.369 , de 8 de julho de 2013. Dispõe sobre a implementação do Projeto Mais Médicos para o Brasil. Diário Oficial da União 2013; 8 jul.

31. Index Mundi. Mapa comparativo entre países: densidade de médicos - mundo. https://www. indexmundi.com/map/?v=2226\&l=pt (acessado em 04/Mar/2019).

32. Oliveira CM, Cruz MM, Kanso S, Reis AC, Lima A, Torres RMC, et al. Avaliabilidade do Programa de Valorização do Profissional da Atenção Básica (PROVAB): desafios para gestão do trabalho. Ciênc Saúde Colet 2015; 20:2999-3010.

33. Pereira LL, Santos LMP, Santos W, Oliveira A, Ratner D. Mais Médicos program: provision of medical doctors in rural, remote and socially vulnerable areas of Brazil, 2013-3014. Rural Remote Health 2016; 16:3616.

34. Oliveira JPA, Sanchez MN, Santos LMP. O Programa Mais Médicos: provimento de médicos em municípios brasileiros prioritários entre 2013 e 2014. Ciênc Saúde Colet 2016; 21:2719-27.

35. Araújo CA, Michelotti FC, Ramos TKS. Government provision programs: profile and motivations of physicians who migrated from the Primary Care Professional Valorization Program (Provab) to the More Doctors Program in 2016. Interface (Botucatu, Online) 2017; 21 Suppl 1:1217-28.

36. Herval AM, Rodrigues ET. Ampliação do acesso e mudança de modelo: experiência a partir do Programa Mais Médicos. Interface (Botucatu, Online) 2016; 21 Suppl 1:1325-32.

37. Mota RG, Barros NF. O Programa Mais Médicos no Estado de Mato Grosso, Brasil: uma análise de implementação. Ciênc Saúde Colet 2016; 21:2879-88.

38. Miranda AS, Melo DA. Análise comparativa sobre a implantação do Programa Mais Médicos em agregados de municípios do Rio Grande do Sul, Brasil. Ciênc Saúde Colet 2016; 21:2837-48

39. Siqueira CEG, Soares GB. Sugestões e críticas dos médicos intercambistas cubanos ao PMM. In: Organização Pan-Americana da Saúde, editor. Interações socioculturais dos médicos cubanos participantes do Programa Mais Médicos no Brasil. Brasília: Organização Pan-Americana da Saúde; 2018. p. 33-55. 


\section{Abstract}

The study aimed to assess the implementation of the More Doctors Program (PMM in Portuguese) based on a case study of the Espirito Santo State, Brazil. The first stage involved analyzing the documents that launched the PMM, allowing the creation of a logic model. In the second stage, data on the PMM in Espirito Santo State were analyzed (2013-2016) based on population brackets, creating indicators for the proportion of physicians before and after the Program with the following parameters: unacceptable (0 to 0.99 physicians / 1,000 inhabitants); acceptable (1 to 1.99); and desirable (2.0 or more). Data were analyzed with the Wilcoxon and Kruskal-Wallis non-parametric tests. The model included the size, inputs, activities, outputs, results, and impact of the PMM, orienting analysis of the Program's implementation. Of all the physicians in the emergency supply to Espirito Santo State (432), the majority (63.8\%) were cooperative exchange physicians (Cubans), while $31.2 \%$ were regional board-accredited Brazilians. The Brazilian and individual exchange physicians were allocated mainly in medium and large municipalities, while the Cubans were distributed more heterogeneously in municipalities of all population sizes and were thus more common in areas with greater social vulnerability and lower social capital. There was a significant increase in the number of physicians in Espirito Santo State, but the supply to the extremely poor population did not reach the desired level, presenting an unacceptable mean rate in municipalities with up to 10,000 inhabitants and an acceptable rate in $\mathrm{mu}$ nicipalities in the other population brackets.

Family Practice; Unified Health System; Primary Health Care; Public Health Policy; Health Evaluation

\section{Resumen}

El objetivo fue evaluar la implantación del programa Más Médicos (PMM), a partir del estudio de caso de Espírito Santo, Brasil. En una primera etapa, se analizaron los documentos que instituyeron el PMM, permitiendo la creación de un modelo lógico. En la segunda etapa, se analizaron datos del PMM en Espírito Santo (2013-2016), a partir de franjas poblacionales, generando la creación de indicadores de la proporción de médicos antes y después del programa con los parámetros: inaceptable ( 0 a 0,99 médicos/1.000 habitantes); aceptable (1 a 1,99); y deseable (2,0 o más). Los datos se analizaron con las pruebas no-paramétricas de Wilcoxon y Kruskal-Wallis. El modelo incluyó dimensiones, insumos, actividades, productos, resultados e impacto del PMM y orientó el análisis de la implantación del Programa. Del total de médicos para la provisión de emergencia para Espírito Santo (432), la mayoría $(63,8 \%)$ pertenecian al perfil intercambios de cooperación (cubanos) y $31,2 \%$ a médicos brasileños registrados en el Consejo Regional de Medicina. Tanto los médicos brasileños, como los intercambios individuales, se ubicaron prioritariamente en municipios de tamaño medio y grande, mientras que los cubanos se distribuyeron de forma más heterogénea en municipios de todos los tamaños poblacionales y, consecuentemente, son más frecuentes en áreas de mayor vulnerabilidad social y menor capital social. Hubo un significativo incremento de la cantidad de médicos en Espírito Santo, no obstante, la provisión de médicos para la población en extrema pobreza no mostró un indicador deseable, presentando una media todavía inaceptable en municipios de hasta 10.000 habitantes y aceptable en los municipios de las demás franjas poblacionales.

Medicina Familiar y Comunitária; Sistema Único de Salud; Atención Primaria de Salud; Políticas Públicas de Salud; Evaluación en Salud
Recebido em 19/Mar/2019

Versão final reapresentada em 29/Out/2019

Aprovado em 11/Nov/2019 